\title{
A Design of Microstrip Array Antenna for ETC Applications
}

\author{
Rui $\mathrm{Li}^{1}$, a Ming Bai ${ }^{1}$, Chun-xiang $\mathrm{Li}^{1}, \mathrm{Hu} \mathrm{Xu}^{1}$, Yi-dan $\mathrm{Su}^{1}$ \\ ${ }^{1}$ Guangzhou Maritime Institute, China \\ No.101 Hongshan Three Road, Huangpu District, Guangzhou 510725 \\ a18620022630@163.com
}

Keywords: ETC, Microstrip Array antenna, Series-fed.

Abstract. A design of microstrip array antenna for ETC (Electronic Toll Collection) applications is presented. The antenna is designed and realized at the center frequency of $5.835 \mathrm{GHz}$, and consists of 32 microstrip patches $(4 \times 8)$. Furthermore, the proposed antenna is series-fed, so it is easy to manufacture and the cost is relatively low. The simulated and measured results agree very well.

\section{Introduction}

In recent years, an enormous growth of road traffic has occurred. In order to reduce more and more traffic congestion, rising number of accidents and more environmental pollution, several research programs in Europe, USA and Japan are launched for an Intelligent Transportation System (ITS). These systems need small size antennas with relatively high gain, narrow main lobe and low side-lobe suppression. Some array antennas (such as antenna with $3 \times 8$ elements) have wide main lobe, and slightly worse sidelobe suppression. The type of problem has been discussed quite a lot from the analytic approaches. And more general numerical approaches have been mentioned in the recent paper ${ }^{[1][2]}$, it seems impossible to make a list in detail. Traditional Taylor Amplitude Distribution is chosen for its low sidelobe characteristic. Tuning is used to maintain beam pattern with equal sidelobe level. To further reduce the array antenna sidelobe level, it can be achieved by changing the radiation element on the current phase, the elements spacing and amplitude distribution. The transmission coefficient between antenna elements in the series array is hard to calculate accurately ${ }^{[3]}$, so simulation and numerical optimization are important in the design. We introduce the antenna with 32 radiation elements $(4 \times 8)$ in this paper. The antenna is series-fed and fabricated on the FR4 substrate with high dielectric constant 3.58 and height of $0.75 \mathrm{~mm}$. To keep the design process simple, array feed lines are split into two symmetric parts. Resonant array is chosen for its radiation band. Circular polarization and diversity techniques help to improve the communication behavior. The proposed antenna is simulated with HFSS software.

\section{Antenna Design}

In order to meet the design requirements, the corner-cut rectangular antenna element is designed to realize right-hand circular polarization. The way for the side-fed is easy to implement the composition of the array. The T-type power splitters and phasing lines are used to realize the wanted excitation as described in ${ }^{[5]}$. In general, Cosine Amplitude Distribution, Chebyshev or Taylor Amplitude Distribution are applied for realizing the low sidelobe level in array antennas design.

In this case, Taylor Amplitude distribution with a $20 \mathrm{~dB}$ weight vector is chosen to get a low sidelobe array pattern, and the Taylor weight vector is as follows:

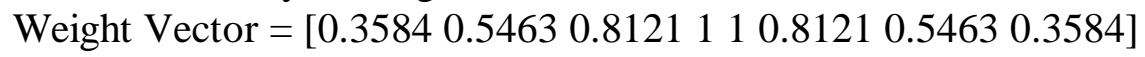

As well known, the sidelobe level depends on the feed lines, array spacing and the pattern of antenna element. To increase the sidelobe suppression level, the weight vector needs slightly tuning. After calculation and optimization, the configuration of the proposed array antenna, through commissioning and optimization, is designed as depicted in Fig. 1. 


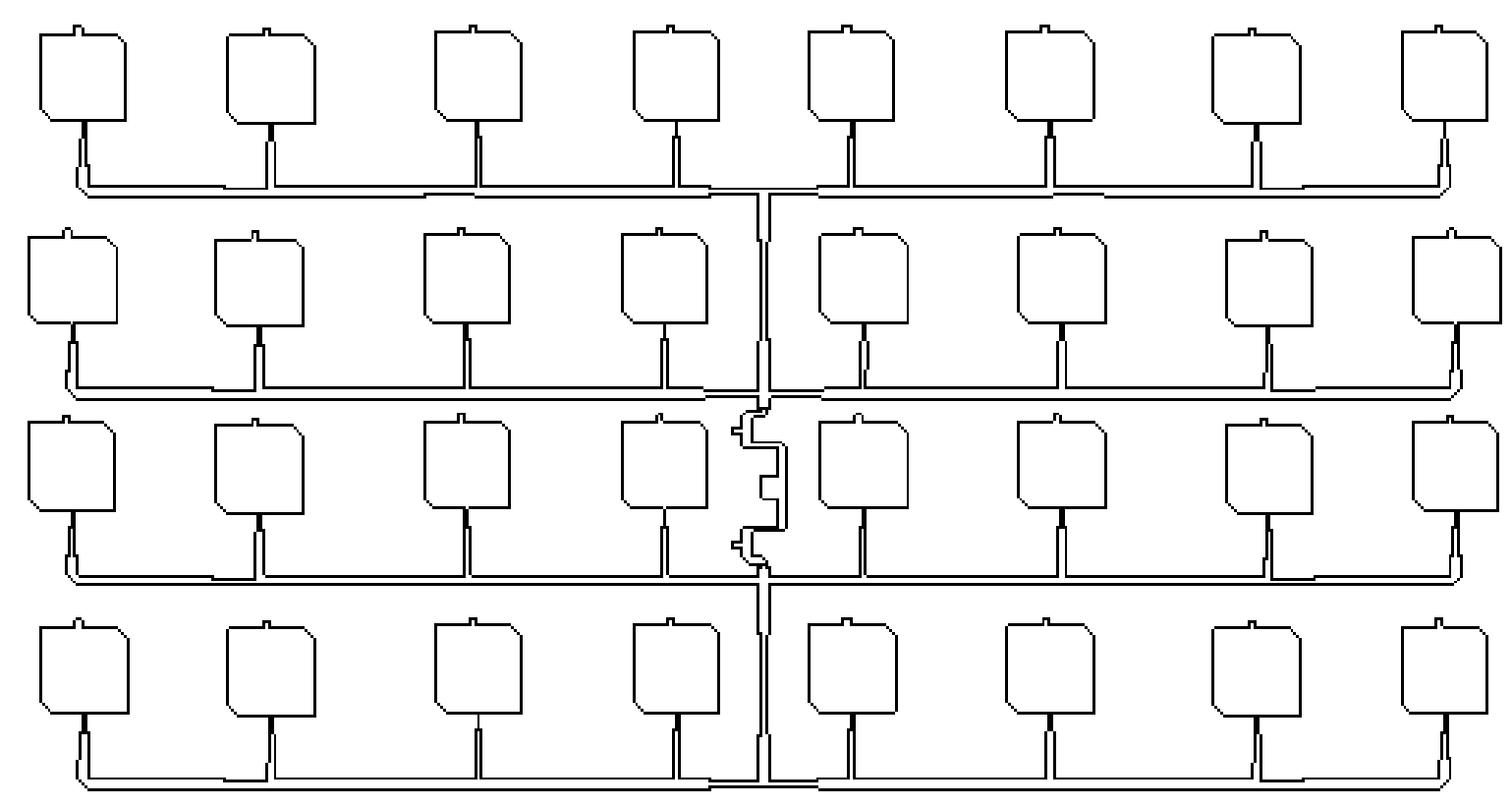

Fig. 1 Design pattern of the proposed array antenna.

\section{Analysis of the Simulation and Measurement Results}

To validate the proposed design, an array antenna with $4 \times 8$ array-elements has been designed, manufactured and measured with the ground plane dimensions of $170 \times 240 \mathrm{~mm}^{2}$ based on FR4 laminate. Each array-element is in the size of $13.12 \times 13.12 \mathrm{~mm}$. Optimizing to maintain a low side-lobe suppression, the distance between the patches is not the same, most of them are spaced $31 \mathrm{~mm}$ more or less apart. The calculated and measured far-field radiation pattern is shown in Fig .2.

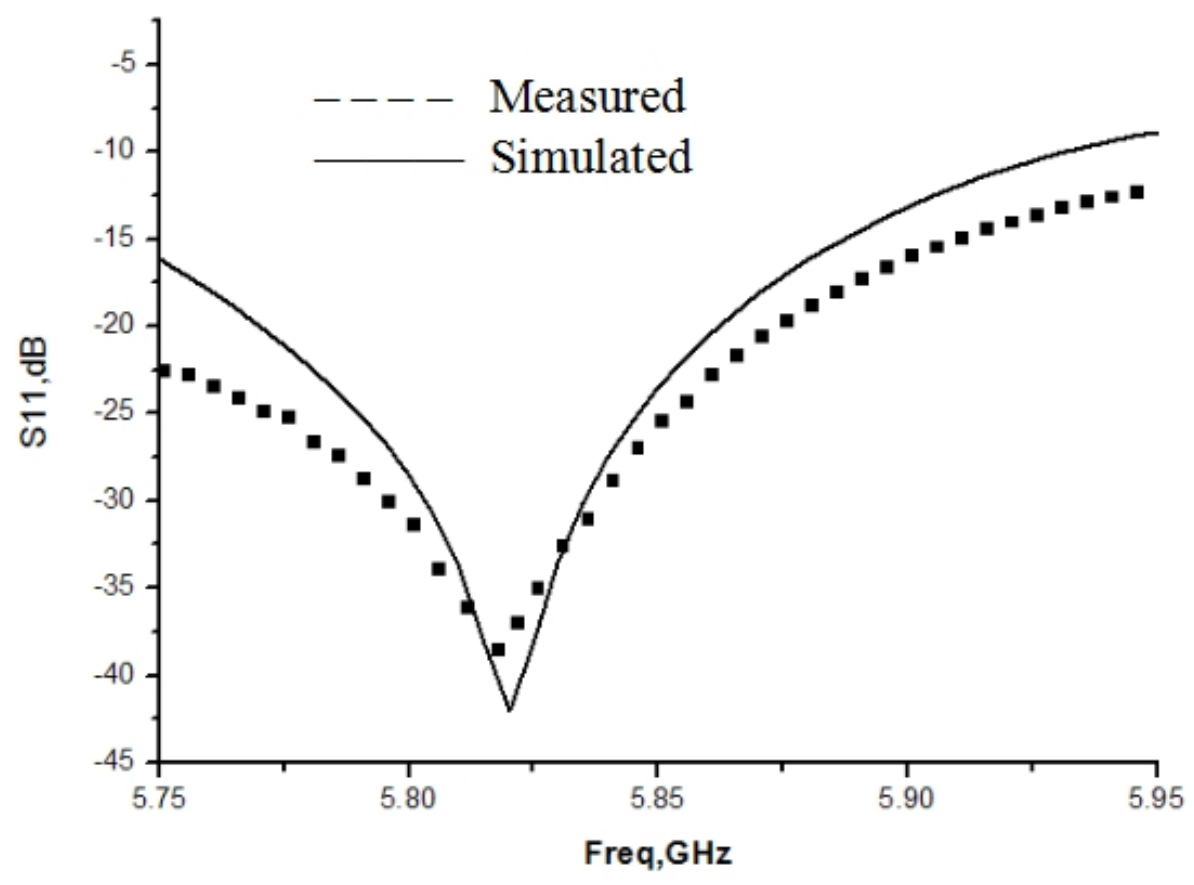

Fig .2 Return losses.

As depicted in Fig.2, the designed antenna has a good characteristic of return losses better than $30 \mathrm{~dB}$. The measured results are slightly different from the calculated ones which are most likely caused by an 
influence of the manufacture deviation and measurement fixtures. The calculated and measured gains are shown in Fig.3.

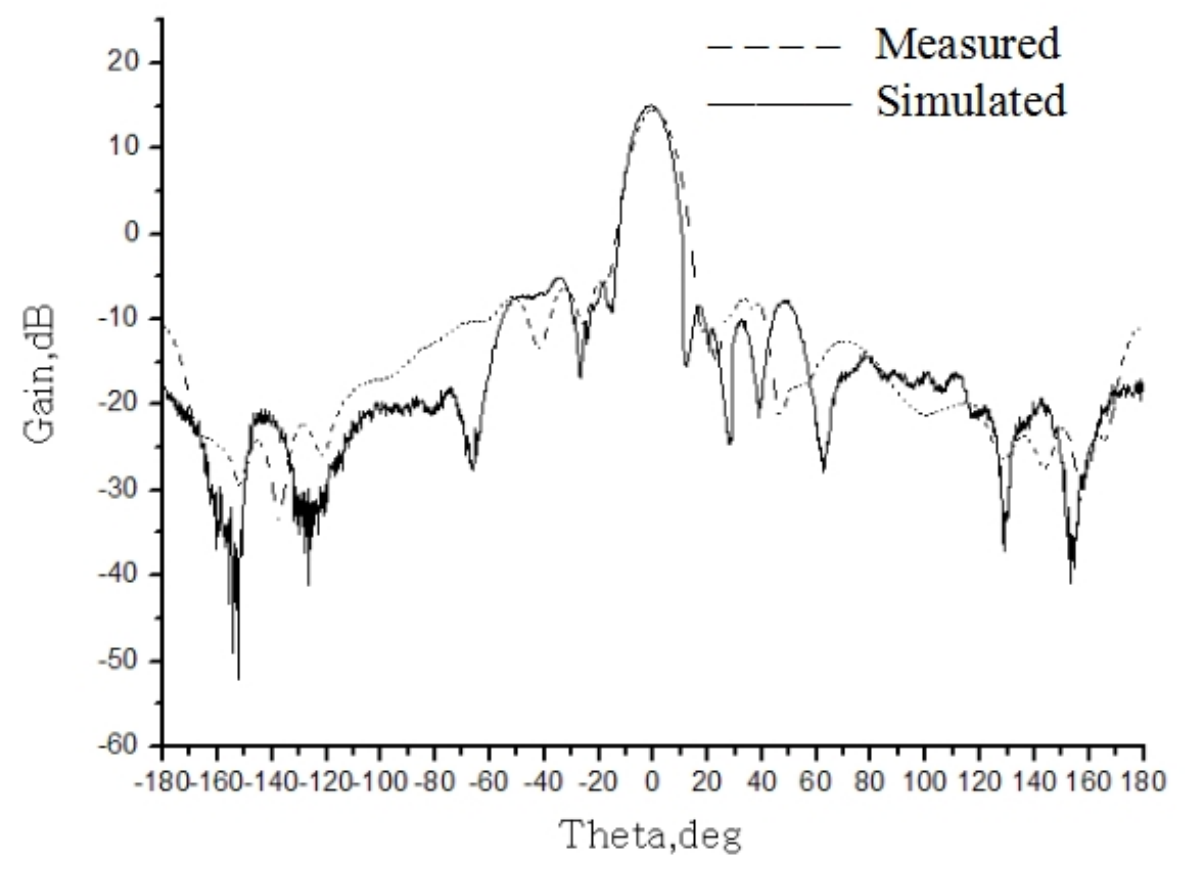

Fig .3 Far-field radiation pattern.

The main sidelobe level suppression is just below $-18 \mathrm{~dB}$ and achieved gain is better than $14.5 \mathrm{dBi}$ within the operational frequency band. This kind of array antenna can fit the requirements of ETC system. The comparison of the measured and simulated results show a good agreement in the main beam direction. The difference between the calculated and measured value is due to the assuming lossless dielectric substrate. Simulation and measurement of the Axis Ratio is also of good agreement as shown in Fig. 4. The Axis Ratio value is below $3 \mathrm{~dB}$ from $-10^{\circ}$ to $10^{\circ}$, it can achieve good right hand circular polarization.

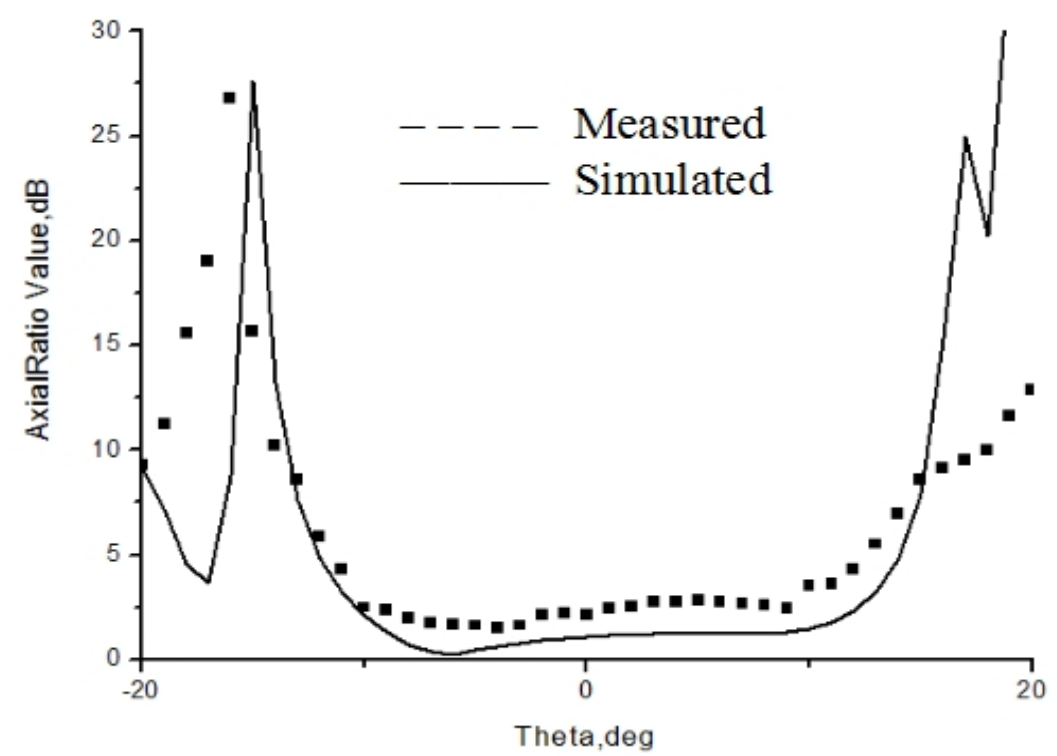

Fig. 4 Axial Ratio for right-circularly 


\section{Conclusion}

A low sidelobe array antenna for ETC system at C band is designed, simulated, and measured. The design method of the proposed antenna is effective, which is also suitable for applications in other bands by tuning the parameters. Array synthesis theory, numerical calculation method and electromagnetic simulation work well together.

\section{Acknowledgments}

This work was supported by the Guangdong Public Welfare Research and Capacity Building Foundation (2015A010103024) and the Innovation and Strong School of Guangdong Province (A510630, A330105).

\section{References}

[1] O.M. Bucci, D. D'Elia, G. Mazzarella, and G. Panatiello: Antenna pattern synthesis: A new general approach, Proceedings of the IEEE, Vol. 82, pp. 358-371, March 1994.

[2] Harry L. Van Trees, Optimum Array Processing(Part IV), John Wiley \& Sons,2002.

[3] K. R. Carver, and J. W. Mink, Microstrip antenna technology, IEEE Trans. Antennas Propagation, Vol. 29, No.1, pp.2-24, Jan. 1981.

[4] J. R. James, P. S. Hall, C. Wood, Microstrip antenna theory and design. P.S.Hall and C. Wood. 1981. 